\title{
Semi-blind Channel Estimation for MIMO-OFDM Systems Based on Received Signal Reconstruction
}

\author{
Weijia Cui ${ }^{1}$, You Zhou ${ }^{2, \text { a }}$, Song Chen ${ }^{3, \mathrm{~b},{ }^{*}}$ \\ ${ }^{1}$ Information Science and Technology Institute, Zhengzhou, 450001, China \\ ${ }^{2}$ Information Science and Technology Institute, Zhengzhou, 450001, China \\ ${ }^{3}$ Information Science and Technology Institute, Zhengzhou, 450001, China \\ a309732242@qq.com, ${ }^{b}$ wirelessmancs@126.com, *Corresponding author
}

\begin{abstract}
Keywords: MIMO-OFDM; Blind channel estimation; Subspace; Signal reconstruction; QR decomposition; Gram-Schmidt orthogonalization;

Abstract. Blind channel estimation for MIMO-OFDM systems was discussed, and a subspace based blind channel estimation algorithm by re-constructing the received signal and its simplified one were proposed. The algorithm changed the channel matrix of the transfer function into a block Toeplitz high matrix exploiting OFDM cyclic prefix, and the channel estimation characteristic equation was obtained with the orthogonality between the signal subspace and the noise subspace of the reconstructed signal. In order to eliminate the influence of virtual carriers, the singular value decomposition of the equivalent transmit signal was imported. The simplified algorithm exploited a QR decomposition and Gram-Schmidt orthogonalization process aiming at reducing the complexity of obtaining the noise subspace. Simulation results illustrate the performance of the proposed algorithm via numerical experiments compared with the other one, and it's insensitive to overestimates of the true channel order.
\end{abstract}

\section{Introduction}

Channel estimation the pre-condition of coherent detection in MIMO-OFDM systems. Traditional method is to insert pilot in the transmitting data [1]. In order to improve frequency spectrum efficiency, blind channel estimation has become hot topic in recent years [2] [7]. Among those blind algorithm, subspace based algorithm receives great attention because of its better accuracy performance [8].

An OFDM subspace algorithm exploiting CP is proposed in [9]. Although with a good accuracy performance, it is not applicable to systems with existence of virtual sub-carriers. Traditional OFDM subspace based algorithm is expanded to MIMO-OFDM systems in [10] with slow convergence rate.

The paper pays attention to fast semi-blind channel estimation in MIMO-OFDM systems with existence of virtual sub-carriers. Learnt from [9], a semi-blind algorithm based on received signal reconstruction and its simplified one are proposed.

Some notations are illustrated as follows: $(\cdot)^{\mathrm{T}},(\cdot)^{*}$ and $(\cdot)^{\mathrm{H}}$ are transposition, conjugate and conjugate transpose respectively. $\mathbf{I}_{N}$ is $N \times N$ unit matrix. $E[\cdot]$ is statistical average. $\mathbf{x}[1: k]$ is the front $k$ elements in $\mathbf{x} . \mathbf{X}[:, k]$ and $\mathbf{X}[k,:]$ are the $k$-th column and $k$-th row of $\mathbf{X} . \mathbf{X}[i: j, m: n]$ is the sub-matrix of $\mathbf{X}$ from row $i$ to $j$ and column $m$ to $n$. $\operatorname{span}(\mathbf{X})$ and $\operatorname{rank}(\mathbf{X})$ are the column space of matrix $\mathbf{X}$ and its rank respectively. $\operatorname{vec}(\mathbf{X})$ is vectorization of matrix $\mathbf{X} \cdot\|\cdot\|_{2}$ is $l_{2}$ norm. $\otimes$ is Kronecker product. Define $w_{N} \square \exp (j 2 \pi / N) . \mathcal{C N}\left(0, \sigma^{2}\right)$ is complex Gaussian distribution.

\section{MIMO-OFDM System Model}

Assume that the number of transmitting and receiving antennas in MIMO-OFDM system are $N_{t}$ and $N_{r}$ respectively with $N_{r} \geq N_{t}$. There are $N$ sub-carriers with $D$ data sub-carriers and $(N-D)$ virtual sub-carriers. The indices of data sub-carriers are denoted by $k_{0}$ to $k_{0}+D-1$. CP and OFDM length are $P$ and $Q=N+P$ 
respectively. Data to be transmitted is $\mathbf{x}(n, k)=\left[x_{1}(n, k), x_{2}(n, k), \ldots, x_{N_{t}}(n, k)\right]^{\mathrm{T}}$, where $x_{i}(n, k)$ is the data on the $k$-th sub-carrier of antenna $i$ in $n$-th OFDM symbol. Accordingly, the total $n$-th data to be modulated can be denoted by $\mathbf{x}_{n}=\left[\mathbf{x}\left(n, k_{0}\right)^{\mathrm{T}}, \mathbf{x}\left(n, k_{0}+1\right)^{\mathrm{T}}, \ldots, \mathbf{x}\left(n, k_{0}+D-1\right)^{\mathrm{T}}\right]^{\mathrm{T}} . \mathbf{s}_{n}$ is the modulated data after insertion of CP. Define $\mathbf{s}(n, k)=\left[s_{1}(n, k), s_{2}(n, k), \ldots, s_{N_{t}}(n, k)\right]^{\mathrm{T}}$, where $s_{i}(n, k)$ the $k$-th data after IFFT on antenna $i$. We have $\mathbf{s}_{n}=\left[\mathbf{s}(n, N-P)^{\mathrm{T}}, \ldots, \mathbf{s}(n, N-1)^{\mathrm{T}}, \mathbf{s}(n, 0)^{\mathrm{T}}, \mathbf{s}(n, 1)^{\mathrm{T}}, \ldots, \mathbf{s}(n, N-1)^{\mathrm{T}}\right]^{\mathrm{T}}$. Define:

$$
\begin{aligned}
\mathbf{F}(i) & =\frac{1}{\sqrt{N}}\left[w_{N}^{k_{0} i}, w_{N}^{\left(k_{0}+1\right) i}, \ldots, w_{N}^{\left(k_{0}+D-1\right) i}\right] \\
\mathbf{F} & =\left[\mathbf{F}(N-P)^{\mathrm{T}}, \ldots, \mathbf{F}(N-1)^{\mathrm{T}}, \mathbf{F}(0)^{\mathrm{T}}, \mathbf{F}(1)^{\mathrm{T}}, \ldots, \mathbf{F}(N-1)^{\mathrm{T}}\right]^{\mathrm{T}} \\
\mathbf{\Gamma} & =\mathbf{F} \otimes \mathbf{I}_{N_{t}}
\end{aligned}
$$

So we have $\mathbf{s}_{n}=\boldsymbol{\Gamma} \mathbf{x}_{n}$. Channel model between transmitting and receiving antennas is denoted by FIR filter, and $L$ is maximum channel order. The $l$-th channel coefficient is denoted by

$$
\mathbf{h}(l)=\left[\begin{array}{cccc}
h_{11}(l) & h_{12}(l) & \cdots & h_{1 N_{t}}(l) \\
h_{21}(l) & h_{22}(l) & \cdots & h_{2 N_{t}}(l) \\
\vdots & \vdots & \vdots & \vdots \\
h_{N_{r} 1}(l) & h_{N_{r} 2}(l) & \cdots & h_{N_{r} N_{t}}(l)
\end{array}\right]
$$

We take $\mathbf{r}_{n}$ as the received signal. Define $\mathbf{r}(n, k)=\left[r_{1}(n, k), r_{2}(n, k), \ldots, r_{N_{r}}(n, k)\right]^{\mathrm{T}}$, where $r_{j}(n, k)$ is the $k$-th received data on receiving antenna $j$ in $n$-th symbol. Accordingly, we have $\mathbf{r}_{n}=\left[\mathbf{r}(n, 0)^{\mathrm{T}}, \ldots, \mathbf{r}(n, 1)^{\mathrm{T}}, \ldots, \mathbf{r}(n, N+P-1)^{\mathrm{T}}\right]^{\mathrm{T}}$. Assume that $L \leq P$, the channel to be estimated is denoted by $\mathbf{h}=[\mathbf{h}(0), \mathbf{h}(1), \ldots, \mathbf{h}(P)]$. When $i>L, \mathbf{h}(i)=\mathbf{0}$. Define

$\mathbf{H}_{0}=\left[\begin{array}{cccc}\mathbf{h}(0) & \mathbf{0} & \mathbf{0} & \mathbf{0} \\ \vdots & \ddots & \mathbf{0} & \mathbf{0} \\ \mathbf{h}(P) & \ddots & \ddots & \mathbf{0} \\ \mathbf{0} & \mathbf{h}(P) & \cdots & \mathbf{h}(0)\end{array}\right], \mathbf{H}_{1}=\left[\begin{array}{cccc}\mathbf{0} & \mathbf{h}(P) & \cdots & \mathbf{h}(1) \\ \vdots & \mathbf{0} & \ddots & \vdots \\ \mathbf{0} & \mathbf{0} & \mathbf{0} & \mathbf{h}(P) \\ \mathbf{0} & \mathbf{0} & \mathbf{0} & \mathbf{0}\end{array}\right]$

we have $\mathbf{r}_{n}=\mathbf{H}_{0} \mathbf{s}_{n}+\mathbf{H}_{1} \mathbf{s}_{n-1}+\mathbf{v}_{n}$, where $\mathbf{v}_{n} \sim \mathcal{C N}\left(\mathbf{0}, \sigma_{v}^{2} \mathbf{I}_{Q}\right)$ is the complex white Guassian noise.

\section{Subspace Algorithm based on Received Signal Reconstruction}

\section{A. Algorithm Deduction}

Devide $\mathbf{r}_{n}, \mathbf{s}_{n}$ and $\mathbf{v}_{n}$ into 3 sub-vectors: $\mathbf{r}_{n}=\left[\mathbf{r}_{n 1}^{\mathrm{T}}, \mathbf{r}_{n 2}^{\mathrm{T}}, \mathbf{r}_{n 3}^{\mathrm{T}}\right]^{\mathrm{T}}, \mathbf{s}_{n}=\left[\mathbf{s}_{n 1}^{\mathrm{T}}, \mathbf{s}_{n 2}^{\mathrm{T}}, \mathbf{s}_{n 3}^{\mathrm{T}}\right]^{\mathrm{T}}, \mathbf{v}_{n}=\left[\mathbf{v}_{n 1}^{\mathrm{T}}, \mathbf{v}_{n 2}^{\mathrm{T}}, \mathbf{v}_{n 3}^{\mathrm{T}}\right]^{\mathrm{T}}$. For $\mathbf{r}_{n}$ and $\mathbf{v}_{n}$, the dimension of the $1^{\text {st }}$ and the $3^{\text {rd }}$ sub-vector is $P N_{r} \times 1$, while for $\mathbf{s}_{n}$, the dimension is $P N_{t} \times 1$. Define $\mathbf{p}_{1}(n)=\left[\mathbf{r}_{(n-1) 2}^{\mathrm{T}}, \mathbf{r}_{(n-1) 3}^{\mathrm{T}}, \mathbf{r}_{n 1}^{\mathrm{T}}\right]^{\mathrm{T}}, \mathbf{p}_{2}(n)=\left[\mathbf{r}_{n 1}^{\mathrm{T}}, \mathbf{r}_{n 2}^{\mathrm{T}}, \mathbf{r}_{n 3}^{\mathrm{T}}\right]^{\mathrm{T}}$, and reconstruct the received signal according to $\mathbf{p}(n)=\mathbf{p}_{1}(n)-\mathbf{p}_{2}(n)$. Exploiting CP characteristics, we have $\mathbf{p}(n)=\widehat{\mathbf{H}} \widehat{\mathbf{s}}(n)+\widehat{\mathbf{v}}(n)$, where $\widehat{\mathbf{H}}, \widehat{\mathbf{s}}(n)$ and $\widehat{\mathbf{v}}(n)$ are denoted as follows:

$$
\widehat{\mathbf{H}}=\left[\begin{array}{ccc}
\mathbf{h}(0) & \mathbf{0} & \mathbf{0} \\
\vdots & \ddots & \mathbf{0} \\
\mathbf{h}(P) & \ddots & \mathbf{h}(0) \\
\mathbf{0} & \ddots & \vdots \\
\mathbf{0} & \mathbf{0} & \mathbf{h}(P)
\end{array}\right], \widehat{\mathbf{s}}(n)=\left[\begin{array}{c}
\mathbf{s}_{(n-1) 2} \\
\mathbf{s}_{(n-1) 3}
\end{array}\right]-\left[\begin{array}{c}
\mathbf{s}_{n 1} \\
\mathbf{s}_{n 2}
\end{array}\right], \widehat{\mathbf{v}}(n)=\left[\begin{array}{c}
\mathbf{v}_{(n-1) 2} \\
\mathbf{v}_{(n-1) 3} \\
\mathbf{v}_{n 1}
\end{array}\right]-\left[\begin{array}{c}
\mathbf{v}_{n 1} \\
\mathbf{v}_{n 2} \\
\mathbf{v}_{n 3}
\end{array}\right]
$$


Apparently, $\hat{\mathbf{H}}$ is a $Q N_{r} \times N N_{t}$ full column rank Toeplitz high matrix with $\operatorname{rank}(\mathbf{h}(0))=N_{t}$. $\mathbf{R}_{\mathbf{p}}=E\left[\mathbf{p}(n) \mathbf{p}(n)^{\mathrm{H}}\right], \mathbf{R}_{\widehat{\mathbf{s}}}=E\left[\widehat{\mathbf{s}}(n) \widehat{\mathbf{s}}(n)^{\mathrm{H}}\right]$ and $\mathbf{R}_{\widehat{\mathbf{v}}}=E\left[\widehat{\mathbf{v}}(n) \widehat{\mathbf{v}}(n)^{\mathrm{H}}\right]$ are covariance matrixes of $\mathbf{p}(n), \widehat{\mathbf{s}}(n)$ and $\widehat{\mathbf{v}}(n)$ respectively. Assume that the noise and transmitting signal are mutual independent with each other, we have

$$
\mathbf{R}_{\mathbf{p}}=\hat{\mathbf{H}} \mathbf{R}_{\hat{\mathbf{s}}} \widehat{\mathbf{H}}^{\mathrm{H}}+\mathbf{R}_{\hat{\mathbf{v}}}
$$

$$
\begin{aligned}
& \text { Define } \\
& \mathbf{F}_{1}=\left[\mathbf{F}(0)^{\mathrm{T}}, \mathbf{F}(1)^{\mathrm{T}}, \ldots, \mathbf{F}(N-1)^{\mathrm{T}}\right]^{\mathrm{T}}
\end{aligned}
$$$$
\mathbf{F}_{2}=\left[\mathbf{F}(N-P)^{\mathrm{T}}, \ldots, \mathbf{F}(N-1)^{\mathrm{T}}, \mathbf{F}(0)^{\mathrm{T}}, \mathbf{F}(1)^{\mathrm{T}}, \ldots, \mathbf{F}(N-P-1)^{\mathrm{T}}\right]^{\mathrm{T}}
$$

We have

$$
\widehat{\mathbf{s}}(n)=\left(\mathbf{F}_{1} \otimes \mathbf{I}_{N_{t}}\right) \mathbf{x}_{n-1}-\left(\mathbf{F}_{2} \otimes \mathbf{I}_{N_{t}}\right) \mathbf{x}_{n}
$$

Assume that all the transmitting data is mutual independent, we have $R_{\mathbf{x}_{n}}=E\left[\mathbf{x}_{n} \mathbf{x}_{n}^{\mathrm{H}}\right]=\mathbf{I}_{D N_{t}}$, then

$$
\mathbf{R}_{\widehat{\mathbf{s}}}=E\left[\widehat{\mathbf{s}}(n) \widehat{\mathbf{s}}(n)^{\mathrm{H}}\right]=\left(\mathbf{F}_{1} \mathbf{F}_{1}^{\mathrm{H}}+\mathbf{F}_{2} \mathbf{F}_{2}^{\mathrm{H}}\right) \otimes \mathbf{I}_{N_{t}}
$$

\section{Do SVD to $\mathbf{R}_{\overline{\mathrm{s}}}$}

$$
\mathbf{R}_{\widehat{\mathbf{s}}}=\mathbf{A} \boldsymbol{\Lambda}_{\widehat{\mathrm{s}}} \mathbf{A}^{H}
$$

Where $\boldsymbol{\Lambda}_{\widehat{\mathrm{s}}}$ is the diagonal matrix composed by non-zero eigenvalues of $\mathbf{R}_{\widehat{\mathrm{s}}}, \mathbf{A}$ is the corresponding eigenvectors. The algorithm is applicable to systems with existence of virtual sub-carriers with this decomposition. $\mathbf{A}$ is a fixed matrix with the same $N, P$ and $N_{t}$, which means only a small amount of additional calculation is required. Moreover,

$$
\mathbf{R}_{\hat{\mathbf{v}}}=E\left[\widehat{\mathbf{v}}(n) \hat{\mathbf{v}}(n)^{\mathrm{H}}\right]=\sigma_{v}^{2}\left[\begin{array}{ccc}
2 \mathbf{I}_{P N_{r}} & \mathbf{0} & -\mathbf{I}_{P N_{r}} \\
\mathbf{0} & 2 \mathbf{I}_{(N-P) N_{r}} & \mathbf{0} \\
-\mathbf{I}_{P N_{r}} & \mathbf{0} & 2 \mathbf{I}_{P N_{r}}
\end{array}\right]
$$

It can be seen that $\widehat{\mathbf{v}}(n)$ is color noise. Apparently, $\mathbf{R}_{\widehat{\mathbf{v}}}$ is a positive definite matrix which can be whitened through Cholesky decomposition $\mathbf{R}_{\hat{\mathbf{v}}}=\sigma_{v}^{2} \mathbf{w} \mathbf{w}^{\mathrm{H}}$, where $\mathbf{w}$ is a lower triangular matrix with positive diagonal elements. Also, $\mathbf{W}^{-1}$ is a fixed matrix with the same $N, P$ and $N_{r}$, which means no real-time calculation of the matrix is required. It can be obtained that

$$
\widehat{\mathbf{R}}_{\mathbf{p}}=\mathbf{w}^{-1} \mathbf{R}_{\mathbf{p}} \mathbf{w}^{-\mathrm{H}}=\left(\mathbf{w}^{-1} \hat{\mathbf{H}} \mathbf{A}\right) \boldsymbol{\Lambda}_{\widehat{\mathbf{s}}}\left(\mathbf{w}^{-1} \widehat{\mathbf{H}} \mathbf{A}\right)^{\mathrm{H}}+\sigma_{v}^{2} \mathbf{I}_{\mathrm{QM}}
$$

$\boldsymbol{\Lambda}_{\widehat{s}}$ is a full rank matrix, and $\operatorname{rank}\left(\mathbf{w}^{-1} \hat{\mathbf{H}} \mathbf{A}\right)=\operatorname{rank}(\hat{\mathbf{H}} \mathbf{A})$. With constraint $\operatorname{rank}(\mathbf{h}(0))=N_{t}$, eigenvalue decompose equation (11)

$$
\widehat{\mathbf{R}}_{\mathbf{p}}=\mathbf{U}_{s} \boldsymbol{\Lambda}_{s} \mathbf{U}_{s}^{\mathrm{H}}+\sigma_{v}^{2} \mathbf{U}_{o} \mathbf{U}_{o}^{\mathrm{H}}
$$

Where the dimension of $\boldsymbol{\Lambda}_{s}, \mathbf{U}_{s}$ and $\mathbf{U}_{o}$ are $D N_{t} \times D N_{t}, Q N_{r} \times D N_{t}$ and $Q N_{r} \times\left(Q N_{r}-D N_{t}\right)$ respectively. $\mathbf{U}_{s}$ and $\mathbf{U}_{o}$ are signal and noise space of $\hat{\mathbf{R}}_{\mathbf{p}}$ respectively. Also

$$
\mathbf{U}_{o}^{\mathrm{H}} \mathbf{w}^{-1} \hat{\mathbf{H}} \mathbf{A}=\mathbf{0}
$$

The above equation is the characteristic equation of the channel estimation.

$$
\text { Define } \mathbf{h}_{i}=\left[\mathbf{h}(0)[:, i]^{\mathrm{T}}, \mathbf{h}(1)[:, i]^{\mathrm{T}}, \ldots, \mathbf{h}(P)[:, i]^{\mathrm{T}}\right]^{\mathrm{T}}, 1 \leq i \leq N_{t}, \tilde{\mathbf{H}}=\left[\mathbf{h}_{1}, \mathbf{h}_{2}, \ldots, \mathbf{h}_{N_{t}}\right] \text { and } \tilde{\mathbf{h}}=\operatorname{vec}\left(\tilde{\mathbf{H}}^{\mathrm{T}}\right) \text {. The }
$$

difference between $\tilde{\mathbf{h}}$ and the actual channel is a invertible constant matrix which can be obtained through insertion of small amount of pilot [12].

\section{B. Algorithm Description}

$\mathbf{R}_{\mathbf{p}}$ is estimated by $\hat{\mathbf{R}}_{\mathbf{p}}=\left(\sum_{n=0}^{N_{b}-1} \mathbf{p}(n) \mathbf{p}(n)^{\mathrm{H}}\right) / N_{b}$, and $\hat{\mathbf{U}}_{o}^{\mathrm{H}}$ is also a estimated matrix. Define cost function $C(\hat{\mathbf{H}})=\sum_{k=1}^{Q N_{r}-D N_{t}}\left\|\hat{\mathbf{V}}[:, k]^{\mathrm{H}} \hat{\mathbf{H}} \mathbf{A}\right\|_{2}^{2}$ with $\hat{\mathbf{V}}=\mathbf{w}^{-\mathrm{H}} \hat{\mathbf{U}}_{o}$. According to equation(13), estimated $\hat{\mathbf{H}}$ is the value which 
can minimize $C(\hat{\mathbf{H}})$. Equally Divide $\hat{\mathbf{V}}[:, k]$ into $Q$ segments and denoted by $\hat{\mathbf{V}}[:, k]=\left[\left(\hat{\mathbf{V}}_{0}^{(k)}\right)^{\mathrm{T}},\left(\hat{\mathbf{V}}_{1}^{(k)}\right)^{\mathrm{T}}, \ldots,\left(\hat{\mathbf{V}}_{Q-1}^{(k)}\right)^{\mathrm{T}}\right]^{\mathrm{T}}$. Define the following matrix:

$\boldsymbol{\kappa}_{k}=\left[\begin{array}{cccc}\hat{\mathbf{V}}_{0}^{(k)} & \hat{\mathbf{V}}_{1}^{(k)} & \ldots & \hat{\mathbf{V}}_{Q-P-1}^{(k)} \\ \hat{\mathbf{V}}_{1}^{(k)} & \hat{\mathbf{V}}_{2}^{(k)} & \ldots & \hat{\mathbf{V}}_{Q-P}^{(k)} \\ \vdots & \vdots & \ldots & \vdots \\ \hat{\mathbf{V}}_{P}^{(k)} & \hat{\mathbf{V}}_{P+1}^{(k)} & \ldots & \hat{\mathbf{V}}_{Q-1}^{(k)}\end{array}\right]$

$\boldsymbol{\Theta}=\sum_{k=1}^{Q N_{r}-D N_{t}}\left(\boldsymbol{\kappa}_{k}^{*} \otimes \mathbf{I}_{N_{t}}\right) \mathbf{A} \mathbf{A}^{\mathrm{H}}\left(\boldsymbol{\kappa}_{k}^{*} \otimes \mathbf{I}_{N_{t}}\right)^{\mathrm{H}}$

Now, $C(\hat{\mathbf{H}})$ can be rewritten as $C(\hat{\mathbf{H}})=\tilde{\mathbf{h}}^{\mathrm{T}} \boldsymbol{\Theta} \tilde{\mathbf{h}}^{*}$.In order to avoid all zero solution, add a limitation of $\left\|\mathbf{h}_{i}\right\|_{2}^{2}=1$. Define $\breve{\mathbf{h}} \square \tilde{\mathbf{h}}^{*}$, the estimation can be accomplished by $\widehat{\breve{\mathbf{h}}}=\underset{\left\|\mathbf{h}_{i}\right\|_{2}^{2}=1}{\arg \min }\left(\breve{\mathbf{h}}^{\mathrm{H}} \boldsymbol{\Theta} \breve{\mathbf{h}}\right)$, where the estimated $\breve{\mathbf{h}}$ is the linear combination of eigenvectors corresponding to the $N_{t} \times N_{t}$ minimum eigenvalues.

\section{Simplified Algorithm}

Calculate the noise space in a iterative manner. If $\boldsymbol{\Phi}(n)$ is a constant matrix, we have [13]

$\mathbf{T}(n)=\mathbf{\Phi}(n) \mathbf{Q}(n-1), \mathbf{T}(n)=\mathbf{Q}(n) \mathbf{R}(n)$

Finally $\mathbf{Q}(n)$ will converge to the eigenvectors corresponding to the main eigenvalues of $\boldsymbol{\Phi}(n)$. The updating of $\hat{\mathbf{R}}_{\mathbf{p}}$ is accomplished as follows:

$\widehat{\mathbf{R}}_{\mathbf{p}}(n)=\alpha \widehat{\mathbf{R}}_{\mathbf{p}}(n-1)+(1-\alpha) \mathbf{w}^{-1} \mathbf{p}(n) \mathbf{p}(n)^{\mathrm{H}} \mathbf{w}^{-\mathrm{H}}$

Where $\alpha$ is the forgetting factor. Substitute $\boldsymbol{\Phi}(n)$ in equation (15) with $\hat{\mathbf{R}}_{\mathbf{p}}(n)$, we have the following approximation[14]

$\mathbf{T}(n) \approx \alpha \mathbf{T}(n-1)+(1-\alpha) \mathbf{w}^{-1} \mathbf{p}(n)\left(\mathbf{Q}^{\mathrm{H}}(n-1) \mathbf{w}^{-1} \mathbf{p}(n)\right)^{\mathrm{H}}$

And $\mathbf{Q}(n)$ will finally converge to $\mathbf{U}_{s}$. The complexity is about $O\left(Q D N_{r} N_{t}\right)$ [14].

$\mathbf{U}_{o}$ can be calculated by Schmidt orthogonalization exploiting the orthogonality between $\mathbf{U}_{o}$ and $\mathbf{U}_{s}$. Make $\mathbf{U}_{o}(n-1)$ orthogonal to $\mathbf{Q}(n)$ via Schimidt orthogonalization, and the processed vector will be treated as the noise space of the current iteration.

Finally $\mathbf{U}_{o}(n)$ will converge to $\mathbf{U}_{o}$. The complexity is about $O\left(Q N_{r}\left(Q N_{r}-D N_{t}\right)^{2}\right)$.

\section{Simulation and Analysis}

Unless otherwise noted, we choose $N_{t}=2, N_{r}=2, N=16, D=16, P=3, L=3, N_{b}=100$, $h_{i j}(l) \sim \operatorname{CV}\left(0, \sigma_{l}^{2}\right)$ with $\sigma_{l}^{2}=a e^{-l / 10}, l=0, \ldots, L, a$ is power normalization factor[11].

Simulation 1: Performance of the proposed algorithm and algorithm in [10] is compared in Fig.1 choosing $J=2$ in [10]. The accuracy and convergence performance are shown in (a) and (b) respectively. The proposed algorithm has better performance both ways. 


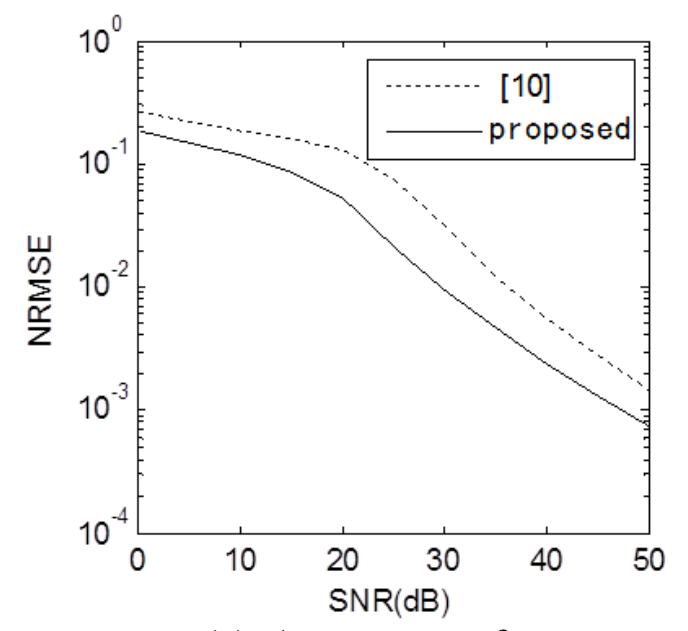

(a) Accuracy performance

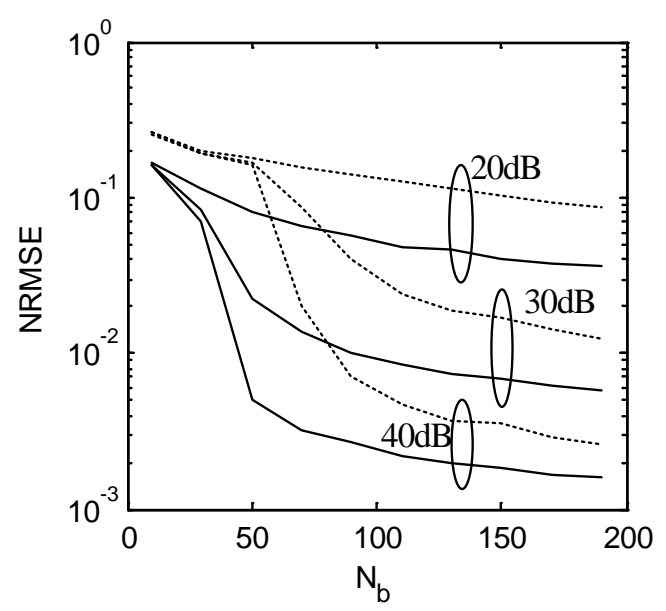

(b) Convergence performance

Fig.1 Performance camparison

Simulation 2: The accuracy performance with different virtual sub-carriers is shown in Fig. 2. We can see that the proposed algorithm can work well with different virtual sub-carriers.

Simulation 3: The accuracy performance of the proposed algorithm with different receiving antennas is shown in Fig.3. The performance improves gradually with the increase of receiving antennas. The reason is that the number of the noise space's basis increases with the increasing of receiving antennas, which means a better anti-interference ability of the proposed algorithm.

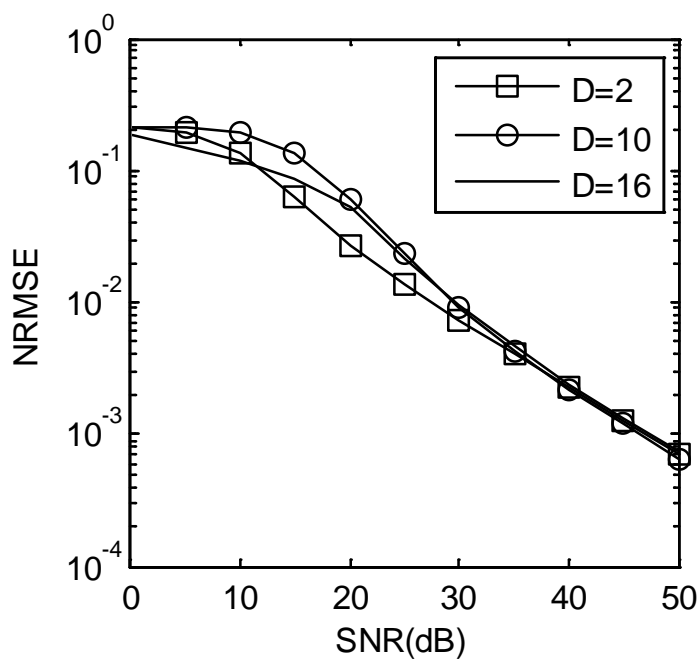

Fig.2 Performance with different virtual subcarriers

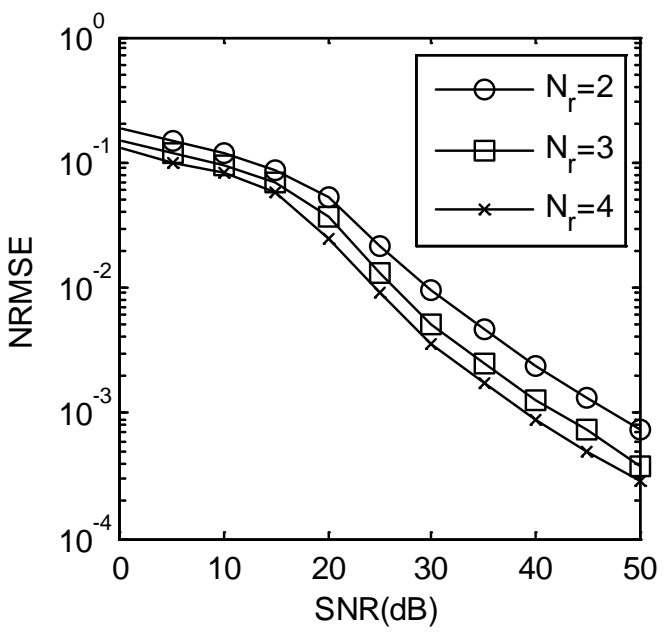

Fig.3 Performance with different receiving antenna

Simulation 4: The accuracy performance with different CP is shown in Fig. 4. We take the CP length as channel order, so the number of channel coefficients to be estimated increases with the increasing of CP. Although the performance degrades slightly with long CP, the algorithm still works well, which means it is robust to channel order overestimation.

Simulation 5: The accuracy performance of simplified algorithm is shown in Fig. 5, where SNR=40 dB, $J=2$. Although the simplified algorithm's accuracy decreases slightly, it still has better performance compared with algorithm in [10]. The complexity of algorithm in [10], SVD and the simplified one are $O(343000), O(54872)$ and $O(2584)$ respectively. 


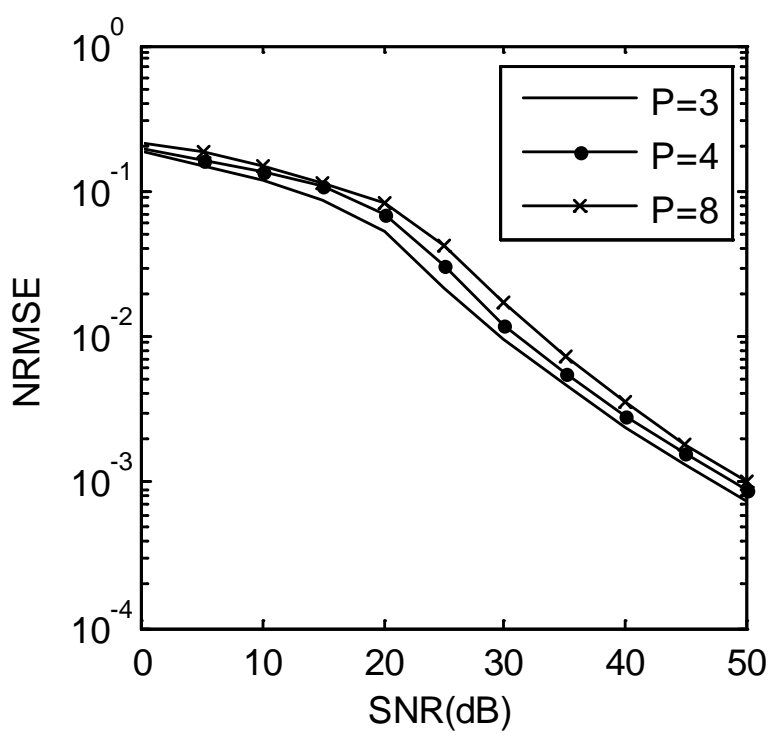

Fig.4 Performance with different CP

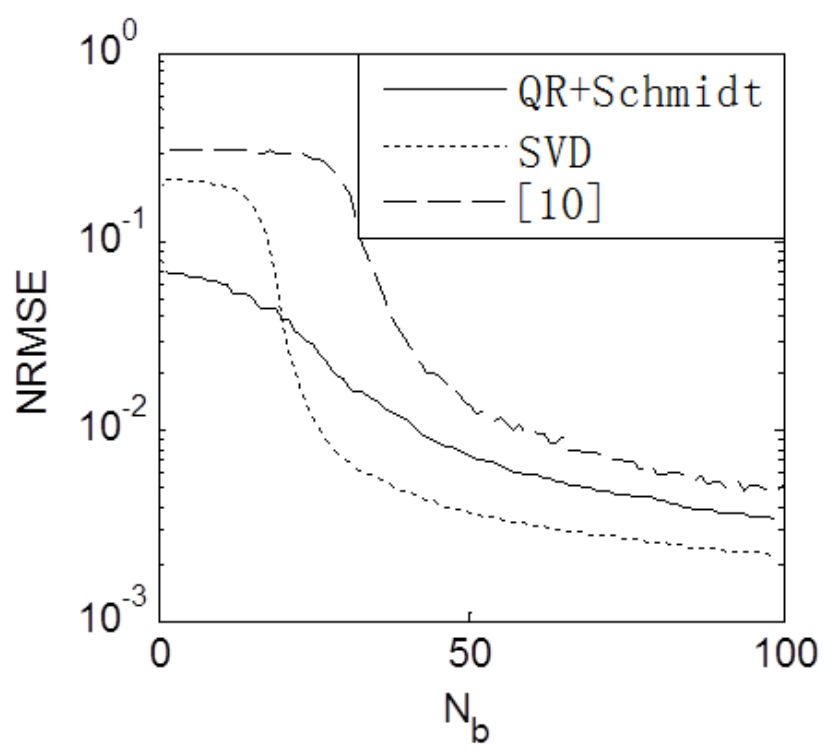

Fig.5 Performance of simplified algorithm

\section{Conclusion}

Exploiting the characteristic of CP, the proposed algorithm reconstructs the received signal and changes the channel matrix into Toeplitz high matrix. Although additional noise whitening and SVD decomposition are required, they are all system parameters related, which means no real-time calculation is needed. The simplified algorithm can effectively reduce the complexity through an iterative method. Simulations show that the proposed algorithm has better accuracy and convergence performance, and is not sensitive to channel order.

\section{References}

[1] Larsen, M.D.. Performance bounds for MIMO-OFDM channel estimation [J]. IEEE Transactions on Signal Processing, 2009, 57(5): 1901-1916.

[2] Miyajima, T., Zhi Ding. Subcarrier nulling algorithms for channel shortening in uplink OFDMA systems [J]. IEEE Transactions on Signal Processing, 2012, 60(5): 2374-2385.

[3] Feng Wan, Wei-Ping Zhu, Swamy, M.N.S.. Semiblind sparse channel estimation for MIMOOFDM systems [J]. IEEE Transactions on Vehicular Technology, 2011, 60(6): 2059-2582.

[4] Al-Naffouri, T.Y., Dahman, A.A., Sohail, M.S., et al.. Low-complexity blind equalization for OFDM systems with general constellations [J]. IEEE Transactions on Signal Processing, 2012, 60(12): 6395-6407.

[5] Al-Bayati, A.K.S.. Subspace-based blind channel estimation in nearly saturated downlink multicarrier code division multiple access systems [J]. IET Communications, 2012, 6(4): 408412.

[6] Zhao Zhi-jin, Wang Bai-chuan, Shang Jun-na, et al.. An estimation algorithm of MIMO-OFDM channels based on RST [J]. Journal of Electronics \& Information Technology, 33(2): 489-493.

[7] Feng Wan, Wei-Ping Zhu, M. N. S. Swamy. Semiblind sparse channel estimation for MIMOOFDM systems [J]. IEEE Transactions on Vehicular Technology, 2011, 60(6): 2569-2582.

[8] Zhang Ling, Zhang Xian-da. An overview of blind channel estimation algorithms for MIMOOFDM systems [J]. ACTA Electronic Sinica, 2007, 35(6A): 1-6.

[9] Huang Xue-jun. Blind channel estimation algorithms in OFDM systems [D]. Shanghai Jiaotong University, 2005. 
[10] Changyong Shin, Robert W. Heath, Jr., et al.. Blind channel estimation for MIMO-OFDM systems [J]. IEEE Transactions on Vehicular Technology, 2007, 56(2): 670-685.

[11] Wei-Chieh Huang, Chun-Hsien Pan, Chih-Peng Li, et al.. Subspace-based semi-blind channel estimation in uplink OFDMA systems [J]. IEEE Transactions on Broadcasting, 2010, 56(1): 58-65.

[12] Y. Zeng, T. S. Ng. A semi-blind channel estimation method for multiuser multiantenna OFDM systems [J]. IEEE Transactions on Signal Processing, 2004, 52(5): 1419-1429.

[13] G. H. Golub, C. F. Van Loan. Matrix Computations, Second edition [M]. Baltimore, MD: John Hopkins University Press, 1989.

[14] Peter Strobach. Low-Rank adaptive filters [J]. IEEE Transactions on Signal Processing, 1996, 44(12): 2932-2947. 